\title{
Structure of Fuzzy Dot d-subalgebras
}

\author{
Gerima Tefera Dejen \\ Department of Mathematics, College of Natural Science, Wollo University, Dessie, Ethiopia.
}

How to cite this paper: Gerima Tefera Dejen. (2020) Structure of Fuzzy Dot d-subalgebras. Journal of Applied Mathematics and Computation, 4(4), 130-136.

DOI: 10.26855/jamc.2020.12.004

Received: August 26, 2020

Accepted: September 22, 2020

Published: October 27, 2020

${ }^{*}$ Corresponding author: Gerima Tefera Dejen, Department of Mathematics, College of Natural Science, WolloUniversity, Dessie, Ethiopia.

Email: gerima233@gmail.com

\begin{abstract}
The concepts of fuzzy dot d-subalgebras, and fuzzy dot d-ideals of a d-algebra have been investigated. The product of fuzzy dot d-ideals and strong fuzzy relation and the corresponding strong fuzzy dot d-ideals has been introduced. Different characterizations are discussed.
\end{abstract}

\section{Keywords}

Fuzzy dot subalgebra, fuzzy d-ideals, fuzzy dot d-ideals, d-algebra, subalgebra

\section{Introduction}

Ahmed [1] introduced the idea of fuzzy BCI-algebra, and Akram and Dar in [2] applied the concept of fuzzy set to d-algebra and they consider the fuzzification of ideals and subalgebra of a d-algebra.

Husein Abbaisa and shukur Al-aeasbi [3] introduced the idea of fuzzy implicative ideal of BH-algebra and different types of fuzzy ideals and fuzzy subsets of BH-algebras and Bormmand seid and etals [4] explained the recent applications of BCK-algebras.

Iseki [5] introduced the notion of ideal theory of a BCK-algebra and Jie Meng, et al. [6] initiated the idea of fuzzy implicative ideals of Bck-algebra. Negger, et al. [7] introduced the concepts of d-ideals in d-algebra, Neggers and Kim [8] introduced the idea of d-algebra which is a generalization of BCK-algebras, and Senapati, et al. [9] discussed the concepts of fuzzy dot ideals of B-algebras. Xi [10] introduced fuzzy BCH-algebras. In 1965, Zadeh [11] initiated the concept of fuzzy subsets. Since then, several researchers have applied this concept to various mathematical disciplines.

In this paper, we introduced the notion of fuzzy dot sub algebras and fuzzy dot d-ideals of a fuzzy d- algebra and we also investigate product of fuzzy dot d-ideals, strong fuzzy dot d-ideals and several basic properties related to fuzzy dot sub algebras and fuzzy dot d-ideals of a fuzzy dot d-algebras.

Throughout this paper, $\mathrm{X}$ represents a d-algebra unless otherwise mentioned.

\section{Methods}

Definition 2.1. [1] An algebra $(X, *, 0)$ of type $(2,0)$ is called a d-algebra, if it satisfies the following axioms:

1. $a * a=0$.

2. $0 * a=0$.

3. $a * b=0$ and $b * a=0 \Rightarrow a=b$, for all a, $\mathrm{b}$ in $\mathrm{X}$.

Let Sbe a non empty subset of a d-algebra X. Then $\mathrm{S}$ is called a subalgebra of X, if $a * b \in S$, for all a, bin $\mathrm{S}$. A subset I of a d-algebra $\mathrm{X}$ is called a $\mathrm{D}$-ideal of $\mathrm{X}$, if it satisfies the following conditions:

1. $0 \in I$. 
2. $a * b \in I$ and $b \in I$ implies $a \in I$.

3. $a \in I, b \in X$, and $a * b \in I$, that is $I \times X \subseteq I$.

Definition 2.2. [1] A mapping $f: X \rightarrow Y$ of a d-algebra is called a homomorphism, if the following hold:

1. $f *(a * b)=f(a) * f(b)$.

2. $f(0)=0^{\prime}$, for all a, b, 0 in $\mathrm{X}$ and $0^{\prime} \in Y$.

Let $\mathrm{X}$ be a non-empty set. A fuzzy subset $\mu$ of the set $\mathrm{X}$ is a mapping $\mu: X \rightarrow[0,1]$.

For a fuzzy subset $\mu$ of a set A. For a fixed $t \in[0,1]$, the set $\mu_{t}=\{a \in X: \mu(a) \geq t\}$ is called an upperlevel of $\mu$.

A fuzzy subset $\mu$ of a d-algebra $\mathrm{X}$ is called a fuzzy sub algebra of $\mathrm{X}$ If it satisfies $\mu(a * b) \geq \min \{\mu(a), \mu(b)\}$, for all $\mathrm{a}, \mathrm{b}$ in $\mathrm{X}$ [11].

Example 2.1. [8] Let $X=\{0, a, b\}$ be a set given by the following Cayley table:

\begin{tabular}{|c|c|c|c|}
\hline$*$ & 0 & $\mathrm{a}$ & $\mathrm{b}$ \\
\hline 0 & 0 & 0 & 0 \\
\hline $\mathrm{a}$ & $\mathrm{a}$ & 0 & $\mathrm{~b}$ \\
\hline $\mathrm{b}$ & $\mathrm{b}$ & $\mathrm{b}$ & 0 \\
\hline
\end{tabular}

Then, $(X, *, 0)$ is a d-algebra.

Define a fuzzy subset $\mu: X \rightarrow[0,1]$ by $\mu(0)=0.8, \mu(a)=0.03$, for all $a \neq 0$ in X. Then $\mu(a * b)=\mu(b) \geq 0.03$.

Hence, $\mu(a * b) \geq \min \{\mu(a), \mu(b)\} \geq 0.03$.

Therefore, $\mu$ is a fuzzy subalgebra of $X$.

Proposition 2.1. [11] A fuzzy subset $\mu$ of a d-algebra $X$ is a fuzzy subalgebra of $X$ if and only if for every $t \in[0,1]$, the upper level set $\mu_{t}$ is either empty or a subalgebra of $\mathrm{X}$.

\section{Results}

\subsection{Structure of fuzzy dot d-subalgebras.}

\section{Definition 3.1.}

Let $\mu$ be a fuzzy subset in a d-algebra $\mathrm{X}$. Then, $\mu$ is called fuzzy dot subalgebra of $\mathrm{X}$ if for all a, $\mathrm{b}$ in $\mathrm{X}, \mu(a * b) \geq \min \{\mu(a), \mu(b)\}$, where “.” denotes ordinary multiplication.

\section{Example 3.1.}

Let $X=\{0,1,2,3\}$ be a set with the following table:

\begin{tabular}{|c|c|c|c|c|}
\hline$*$ & 0 & 1 & 2 & 3 \\
\hline 0 & 0 & 0 & 0 & 0 \\
\hline 1 & 2 & 0 & 3 & 3 \\
\hline 2 & 1 & 1 & 0 & 3 \\
\hline 3 & 2 & 2 & 2 & 0 \\
\hline
\end{tabular}

Then, $(X, *, 0)$ is a d-algebra.

Define a fuzzy subset $\mu: X \rightarrow[0,1]$ by $\mu(0)=0.8, \mu(a)=0.2$, for all $a \neq 0$.

It is very simple to show that $\mu$ is a fuzzy dot subalgebra of a d-algebra $\mathrm{X}$.

\section{Proposition 3.1.}

Every fuzzy dot subalgebra $\mu$ of X satisfies the inequality $\mu(0) \geq(\mu(a))^{2}$, for all a in X

\section{Proof.}

Let $\mu$ be a fuzzy dot sub algebra of $\mathrm{X}$, and let $a \in X$ be arbitrary element. Then we have $a * a=0$ by definition of 
d-algebra.

$\mu(0)=\mu(a * a) \geq \mu(a) . \mu(a)=(\mu(a))^{2}$. Since $\mu$ is fuzzy dot subalgebra of X. Hence, $\mu(0) \geq(\mu(a))^{2}$, for all a in X.

\section{Example 3.2.}

Consider a d-algebra $X=\{0, a, b, c\}$ with the following cayley table:

\begin{tabular}{|c|c|c|c|c|}
\hline$*$ & 0 & $\mathrm{a}$ & $\mathrm{b}$ & $\mathrm{c}$ \\
\hline 0 & 0 & 0 & 0 & 0 \\
\hline $\mathrm{a}$ & $\mathrm{a}$ & 0 & 0 & $\mathrm{a}$ \\
\hline $\mathrm{b}$ & $\mathrm{b}$ & $\mathrm{c}$ & 0 & $\mathrm{c}$ \\
\hline $\mathrm{c}$ & $\mathrm{c}$ & 0 & 0 & 0 \\
\hline
\end{tabular}

Then, $(X, *, 0)$ is a d-algebra.

Define a fuzzy subset $\mu$ in X by $\mu(0)=0.6, \mu(a)=0.7, \mu(b)=0.3$ and $\mu(c)=0.2$. It is easy to verify that $\mu$ is a fuzzy dot sub algebra of X.

\section{Remark 3.1.}

Every fuzzy subalgebra is a fuzzy dot subalgebra, but the converse is not true.

$\mu(a * a)=\mu(0)=0.6<0.7=\mu(a) \geq \min \{\mu(a), \mu(a)\}=0.7$.

Hence $\mu$ is not a fuzzy sub algebra of $\mathrm{X}$ by example 3.2.

\section{Corollary 3.1.}

If $\mu$ is a fuzzy dot subalgebra of $\mathrm{X}$, then, $\mu\left(0^{n} * a\right) \geq(\mu(a))^{2 n+1}$, for all a in $\mathrm{X}$, and $\mathrm{n}$ in $\mathrm{N}$, where $0^{n} * a=0 *(0 * 0) * \ldots(0 * a) \ldots$ in which 0 occurs n- times.

\section{Corollary 3.2.}

Let $\mu$ be a fuzzy dot subalgebra of a d-algebra X.

If there exists a sequence $a_{n}$ in X such that $\lim _{n \rightarrow \infty}\left(\mu\left(a_{n}\right)\right)^{n}=1$, then $\mu(0)=1$.

\section{Theorem 3.1.}

If $\mu$ and $\lambda$ are fuzzy dot sub algebras of, then so is $\mu \cap \lambda$.

\section{Proof.}

Let $\mathrm{a}, \mathrm{b}$ in $\mathrm{X}$. Then,

$$
(\mu \cap \lambda)(a * b)=\min \{\mu(a * b), \lambda(a * b)\} \geq \min (\mu(a) \cdot \mu(b), \lambda(a) \cdot \lambda(b)\}
$$$$
\geq \min \{\mu(a), \lambda(a)\} \cdot \min \{\mu(b), \lambda(b)\}=(\mu \cap \lambda)(a) \cdot(\mu \cap \lambda)(b) \text {. }
$$

Therefore, $\mu \cap \lambda$ is a fuzzy dot subalgebra of $X$.

Remark 3.2. A fuzzy subset $\mu$ of $X$ is a fuzzy subalgebra of $X$ if and only if $\mu_{t}=\{a \in X: \mu(a) \geq t\}$ is a subalgebra of $\mathrm{X}$ for every $t \in[0,1]$, But if $\mu$ is a fuzzy dot subalgebra of $X$, then there exists $t \in[0,1]$ such that $\mu_{t}$, for $t \in[0,1]$ is not a subalgebra as in example $3.2 \mu_{0.3}=\{a \in X: \mu(a) \geq 0.3\}=\{0, a, b\}$ is not a subalgebra of X, since $b * a=c \notin \mu_{0.3}$.

\section{Definition3.2.}

Let $\mathrm{B}$ be a non-empty subset of a d-algebra $\mathrm{X}$. Then, the characteristic function $\mathrm{X}_{B}$ is a special fuzzy set. So that $\mathrm{X}_{B}$ is defined by $X_{B}=\left\{\begin{array}{l}1 \text { If } a \in B \\ 0 \text { if } a \notin B\end{array}\right.$.

\section{Theorem 3.2.}

Let $\mathrm{B}$ be a non-empty subset of $\mathrm{X}$. Then $\mathrm{B}$ is a subalgebra of $\mathrm{X}$ if and only if $\mathrm{X}_{B}$ is a fuzzy dot subalgebra of $\mathrm{X}$.

Let $g: X \rightarrow Y$ be a homomorphism of a d-algebra. If $\mu$ is a fuzzy dot subalgebra of $\mathrm{Y}$, then the preimage $\mu^{g}$ of $\mu$ under $\mathrm{g}$ is a fuzzy dot subalgebra of $\mathrm{X}$.

Theorem 3.3.

If $f: X \rightarrow Y$ be an onto homomorphism of a d-algebra. 
If $\mu$ is a fuzzy dot subalgebra of $\mathrm{X}$, then the image $f(\mu)$ of $\mu$ under $\mathrm{f}$ is a fuzzy dot subalgebra of $\mathrm{Y}$.

Proof. Let $b_{1}, b_{2} \in Y$ and let $A_{1}=f^{-1}\left(b_{1}\right), A_{2}=f^{-1}\left(b_{2}\right)$ and $A_{12}=f^{-1}\left(b_{1} * b_{2}\right)$.

Consider the set $A_{1} * A_{2}=\left\{a \in X: a=a_{1} * a_{2}\right\}$ for some $a_{1} \in A_{1}$ and $a_{2} \in A_{2}$.

So that $f(a)=f\left(a_{1} * a_{2}\right)=f\left(a_{1}\right) * f\left(a_{2}\right)=b_{1} * b_{2}$.

Implies $f(a)=b_{1} * b_{2}$. It follows that $a \in f^{-1}\left(b_{1} * b_{2}\right)=A_{12}$.

Hence $A_{1} * A_{2} \subseteq A_{12}$.

$f\left(\mu\left(b_{1} * b_{2}\right)\right)=\sup \mu(a), a \in A_{1} * A_{2} \geq \sup \left(a_{1} * a_{2}\right), a_{1} \in A_{1}, a_{2} \in A_{2}$.

$\geq\left(\sup \mu\left(a_{1}\right), a_{1} \in f^{-1}\left(b_{1}\right)\right)$. (sup $\left.\mu\left(a_{2}\right), a_{2} \in f^{-1}\left(b_{2}\right)\right)=f(\mu)\left(b_{1}\right) \cdot f(\mu)\left(b_{2}\right)$.

Hence $f\left(\mu\left(b_{1} * b_{2}\right) \geq f(\mu)\left(b_{1}\right) . f(\mu)\left(b_{2}\right)\right.$. Hence, $f(\mu)$ is fuzzy dot subalgebra of Y.

\section{Fuzzy dot d-ideals}

\section{Definition 4.1.}

A fuzzy subset $\mu$ in $\mathrm{X}$ is called fuzzy dot d-ideal of a d-algebra $\mathrm{X}$, if it satisfies the following inequalities:

1. $\mu(0) \geq \mu(a)$, for all $a \in X$.

2. $\mu(a) \geq \mu(a * b) \cdot \mu(b)$.

3. $\mu((a * b) \geq \mu(a) \cdot \mu(b)$, for all a, b in $\mathrm{X}$.

\section{Example 4.1.}

Let $X=\{0, a, b, c\}$ be a d-algebra with the following cayley table:

\begin{tabular}{|c|c|c|c|c|}
\hline$*$ & 0 & $\mathrm{a}$ & $\mathrm{b}$ & $\mathrm{c}$ \\
\hline 0 & 0 & 0 & 0 & 0 \\
\hline $\mathrm{a}$ & $\mathrm{a}$ & 0 & 0 & $\mathrm{c}$ \\
\hline $\mathrm{b}$ & $\mathrm{b}$ & $\mathrm{b}$ & 0 & 0 \\
\hline $\mathrm{c}$ & $\mathrm{c}$ & $\mathrm{c}$ & $\mathrm{c}$ & 0 \\
\hline
\end{tabular}

Then, $(X, *, 0)$ is a d-algebra. We define a fuzzy subset $\mu$ in X by $\mu(0)=0.9$ and $\mu(a)=0.02$, for all $a \neq 0$ in X. Then, $\mu(0)=0.9>0.02=\mu(a)$.

Hence, $\mu(0) \geq \mu(a)$, for all $a \in X$.

1. $\mu(a * b)=\mu(0)=0.9 \geq \mu(a) . \mu(b), a, b \neq 0$. Implies $\mu(a * b) \geq \mu(a) . \mu(b)$, for $a * b=0$ and for $\mu(a * b)=0.02$, for all $a * b \neq 0$.

We have $\mu(a * b) \geq \mu(a) . \mu(b)$. Hence, $\mu(a * b) \geq \mu(a)$. $\mu(b)$., for all a, b in X.

2. $\mu(a) \geq \min \{\mu(a * b), \mu(b)\} \geq \mu(a * b) . \mu(b)$, for all a, b in $\mathrm{X}$.

Thus $\mu$ is a fuzzy dot $\mathrm{d}$ - ideal of a d-algebra $\mathrm{X}$.

\section{Theorem 4.1.}

If $\mu$ is a fuzzy ideal of a d-algebra X such that $\mu(a * 0) \geq \mu(a)$, for all a in X, then $\mu$ is a fuzzy dot ideal of X.

Proposition 4.1. Let $\mu$ be a fuzzy dot ideal. If $a \leq b$ in X, then $\mu(a) \geq \mu(0) . \mu(b)$, for all a, b in X.

Proof. Let a, $\mathrm{b}$ in X such that $a \leq b$. Then $a * b=0$.

So that $\mu(a) \geq \mu(a * b) . \mu(b)=\mu(0) . \mu(b)$. Hence $\mu(a) \geq \mu(0) . \mu(b)$.

Theorem 4.2.

Let $\mu$ and $\lambda$ be two fuzzy dot ideal of a d-algebra X. Then $\mu \cap \lambda$ is also a fuzzy dot ideal of a d-algebra X.

Proof. Let $\mu$ and $\lambda$ be two fuzzy dot ideal of a d- algebra X. Then for any $a \in X, \mu(0) \geq \mu(a)$ and $\lambda(0) \geq \lambda(a)$ : 
1. Now

$(\mu \cap \lambda)(0)=\min \{\mu(0) \cdot \lambda(0)\}=\min \{\mu(a * a), \lambda(a * a)\}$

$\geq \min \{\min \{\mu(a), \mu(a)\}, \min \{\lambda(a), \lambda(a)\}\}=\min \{\mu(a), \lambda(a)\}=(\mu \cap \lambda)(a)$.

Hence $(\mu \cap \lambda)(0) \geq(\mu \cap \lambda)(a)$.

2. Again for any $\mathrm{a}, \mathrm{b}$ in $\mathrm{X}$, we have

$(\mu \cap \lambda)(a)=\min \{\mu(a), \lambda(a)\} \geq \min \{\mu(a * b) . \mu(b), \lambda(a * b) . \lambda(b)\}$

$=\min \{\mu(a * b), \lambda(a * b)\} \cdot \min \{\mu(b), \lambda(b)\}=(\mu \cap \lambda)(a * b) \cdot(\mu \cap \lambda)(b)$.

3. $(\mu \cap \lambda)(a * b)=\min \{\mu(a * b), \lambda(a * b)\} \geq \min \{\mu(a) \cdot \mu(b), \lambda(a) . \lambda(b)\}$

$\geq \min \{\mu(a), \lambda(a)\} \cdot \min \{\mu(b), \lambda(b)\}=(\mu \cap \lambda)(a) \cdot(\mu \cap \lambda)(b)$.

Hence, $(\mu \cap \lambda)(a * b) \geq(\mu \cap \lambda)(a) .(\mu \cap \lambda)(b)$, for all a, b in X.

Therefore, $\mu \cap \lambda$ is a fuzzy dot ideal of a d-algebra $\mathrm{X}$.

\section{Proposition 4.2.}

Every fuzzy dot d-ideal of a d- algebra is fuzzy dot d-sub algebra.

Definition 4.2. Let $\mu$ and $\lambda$ be a fuzzy d-ideals of a d-algebra $X$. Then, $(\mu \times \lambda)(a)=\min \{\mu(a), \lambda(a)\}$, for all a in $X$, where $\mu \times \lambda: X \rightarrow[0,1]$.

Theorem 4.2.

If $\mu$ and $\lambda$ are fuzzy dot d-ideals of $X$. Then, $\mu \times \lambda$ is a fuzzy dot d-ideals of $X \times X$.

Proof. Let $(a, b) \in X \times X$. Then we have

1. $(\mu \times \lambda)(0.0)=\min \{\mu(0), \lambda(0)\} \geq \min \{\mu(a), \lambda(b)\}=(\mu \times \lambda)(a, b)$.

Hence $(\mu \times \lambda)(0,0) \geq(\mu \times \lambda)(a, b)$.

1. Let $a_{1}, a_{2}$ and $b_{1}, b_{2}$ be in $X \times X$.

$(\mu \times \lambda)\left(a_{1}, a_{2}\right)=\min \left\{\mu\left(a_{1}\right), \lambda\left(a_{2}\right)\right\} \geq \min \left\{\min \left\{\mu\left(a_{1} * b_{1}\right), \mu\left(b_{1}\right)\right\}, \min \left\{\lambda\left(a_{2} * b_{2}\right), \lambda\left(b_{2}\right)\right\}\right.$

$\geq \min \left\{\mu\left(a_{1} * b_{1}\right), \lambda\left(a_{2} * b_{2}\right)\right\} \cdot \min \left\{\mu\left(b_{1}\right), \lambda\left(b_{2}\right)\right\}=\left((\mu \times \lambda)\left(a_{1} * b_{1}, a_{2} * b_{2}\right),(\mu \times \lambda)\left(b_{1}, b_{2}\right)\right)$,

where $\left(a_{1}, a_{2}\right)$ and $\left(b_{1}, b_{2}\right)$ in $X \times X$.

2. Let $\left(a_{1}, b_{1}\right)$ and $\left(a_{2}, b_{2}\right)$ be in $X \times X$. Then

$(\mu \times \lambda)\left(\left(a_{1}, b_{1}\right) *\left(a_{2}, b_{2}\right)\right)=(\mu \times \lambda)\left(a_{1} * a_{2}, b_{1} * b_{2}\right)=\min \left\{\mu\left(a_{1} * a_{2}\right), \lambda\left(b_{1} * b_{2}\right)\right\}$

$\geq \min \left\{\min \left\{\mu\left(a_{1}\right), \mu\left(a_{2}\right)\right\}, \min \left\{\lambda\left(b_{1}\right), \lambda\left(b_{2}\right)\right\}\right\} \geq \min \left\{\mu\left(a_{1}\right), \mu\left(a_{2}\right), \lambda\left(b_{1}\right), \lambda\left(b_{2}\right)\right\}$

$\geq \min \left\{\mu\left(a_{1}\right), \lambda\left(b_{1}\right)\right\} \cdot \min \left\{\mu\left(a_{2}\right), \lambda\left(b_{2}\right)\right\} \geq(\mu \times \lambda)\left(a_{1}, b_{1}\right) \cdot(\mu \times \lambda)\left(a_{2}, b_{2}\right)$.

Thus, $\mu \times \lambda$ is a fuzzy dot d-ideal of $X \times X$.

Definition 4.3.

Let $\theta$ be a fuzzy subset of a d-algebra $\mathrm{X}$. The strongest fuzzy $\theta$ relation $\mathrm{X}$ is the fuzzy subset $\mu_{\theta}$ of $X \times X$ defined by $\mu_{\theta}(a, b)=\min \{\theta(a), \theta(b)\}$, where $\mu: X \rightarrow[0,1]$.

Theorem 4.3. Let $\theta$ be a fuzzy subset of a d-algebra $\mathrm{X}$ and $\mu_{\theta}$ be the strongest fuzzy relation on. Then, $\theta$ is a fuzzy dot d-ideal of $\mathrm{X}$ if and only if $\mu_{\theta}$ is a fuzzy dot d-ideal of $X \times X$.

Proof. Suppose $\theta$ be a fuzzy dot d-ideal of $\mathrm{X}$. Then,

1. For any, a, $\mathrm{b}$ in $\mathrm{X}$,

$\mu_{\theta}(0,0)=\min \{\theta(0), \theta(0)\} \geq \min \{\theta(a), \theta(b)\} \geq \theta(a) \cdot \theta(b)$.

Hence, $\mu_{\theta}(0,0) \geq \mu_{\theta}(a, b)$ for all $\mathrm{a}, \mathrm{b}$ in $\mathrm{X}$.

2. Let $a=\left(a_{1}, a_{2}\right)$ and $b=\left(b_{1}, b_{2}\right)$ be in $X \times X$.

$\mu_{\theta}(a)=\mu_{\theta}\left(a_{1}, a_{2}\right)=\min \left\{\theta\left(a_{1}\right), \theta\left(a_{2}\right)\right\} \geq \min \left\{\min \left\{\theta\left(a_{1} * b_{1}\right), \theta\left(b_{1}\right)\right\}, \min \left\{\theta\left(a_{2} * b_{2}\right), \theta\left(b_{2}\right)\right\}\right.$

$\geq \min \left\{\theta\left(a_{1} * b_{1}\right), \theta\left(a_{2} * b_{2}\right)\right\} \cdot \min \left\{\theta\left(b_{1}\right), \theta\left(b_{2}\right)\right\}=\mu_{\theta}\left(a_{1} * b_{1}, a_{2} * b_{2}\right) \cdot \mu_{\theta}\left(b_{1}, b_{2}\right)$.

Hence, $\mu_{\theta}(a) \geq \mu_{\theta}\left(a_{1} * b_{1}, a_{2} * b_{2}\right) . \mu_{\theta}\left(b_{1}, b_{2}\right)$. 
3. $\mu_{\theta}\left(\left(a_{1}, a_{2}\right) *\left(b_{1}, b_{2}\right)\right)=\mu_{\theta}\left(a_{1} * b_{1}, a_{2} * b_{2}\right)=\min \left\{\theta\left(a_{1} * b_{1}\right), \theta\left(a_{2} * b_{2}\right)\right\}$

$\geq \min \left\{\theta\left(a_{1}\right), \theta\left(a_{2}\right)\right\} \cdot \min \left\{\theta\left(b_{1}\right), \theta\left(b_{2}\right)\right\}=\mu_{\theta}\left(a_{1}, a_{2}\right) \cdot \mu_{\theta}\left(b_{1}, b_{2}\right)$.

Hence, $\mu_{\theta}(a * b) \geq \mu_{\theta}\left(a_{1}, a_{2}\right) \cdot \mu_{\theta}\left(b_{1}, b_{2}\right)$. Thus, $\mu_{\theta}$ is a fuzzy dot d-ideal of a d-algebra X.

Conversely, suppose $\mu_{\theta}$ be a fuzzy dot d- ideal of a d-algebra X. Then

1. For any $a \in X$., $\theta(0)=\theta(a * a) \geq \min \{\theta(a), \theta(a)\}=\theta(a)$.

Hence, $\theta(0) \geq \theta(a)$, for all $a \in X$.

2. Let $a, b \in X$. Then,

$\theta(a)=\min \{\theta(a), \theta(a)\}=\mu_{\theta}(a)$

$\geq \mu_{\theta}(a * b) \cdot \mu_{\theta}(b)$

since $\mu_{\theta}$ is a fuzzy dot d- ideal of X.

$=\min \{\theta(a * b), \theta(a * b)\} \cdot \min \{\theta(b), \theta(b)\}=\theta(a * b) \cdot \theta(b)$.

Hence, $\theta(a) \geq \theta(a * b) . \theta(b)$, for all $a, b \in X$.

3. $\theta(a * b)=\min \{\theta(a * b), \theta(b)\}=\mu_{\theta}(a * b) \geq \mu_{\theta}(a) \cdot \mu_{\theta}(b)$

$=\min \{\theta(a), \theta(a)\} \cdot \min \{\theta(b), \theta(b)\}=\theta(a) \cdot \theta(b)$.

Hence, $\theta(a * b) \geq \theta(a) . \theta(b)$, for all $a, b \in X$.

Hence, $\theta$ is a fuzzy dot d- ideal of X.

Theorem 4.4.

Let $f: X \rightarrow Y$ be a homomorphism of d-algebras.

If $\mu$ is a fuzzy dot d-ideal of $\mathrm{Y}$, then $\mu^{f}$ is a fuzzy dot d-ideal of $\mathrm{X}$.

Proof. Let $f: X \rightarrow Y$ be a homomorphism of d- algebras and let $\mu$ be a fuzzy dot d-ideal of Y. Then for any $a \in X$, we have

1. $\mu^{f}(a)=\mu(f(a)) \leq \mu\left(0^{\prime}\right)=\mu\left(f(0)=\mu^{f}(0)\right.$.

Hence, $\mu^{f}(0) \geq \mu^{f}(a)$.

2. Let $a, b \in X$. Then $\mu^{f}(a * b) \cdot \mu^{f}(b) \leq \min \left\{\mu^{f}(a * b), \mu^{f}(b)\right\}$

$$
=\min \{\mu(f(a * b)), \mu(f(b))\} \leq \mu(f(a))=\mu^{f}(a) .
$$

Hence, $\mu^{f}(a) \geq \mu^{f}(a * b) \cdot \mu^{f}(b)$.

3. Let $a, b \in X$. Then $\begin{aligned} \mu^{f}(a) \cdot \mu^{f}(b) \leq \min \left\{\mu^{f}(a), \mu^{f}(b)\right\}=\min \{\mu(f(a), \mu(f(b))\} \\ \leq \mu(f(a) * f(b))=\mu(f(a * b))=\mu^{f}(a * b) .\end{aligned}$

Hence, $\mu^{f}(a * b) \geq \mu^{f}(a) \cdot \mu^{f}(b)$.

Hence, $\mu^{f}$ is a fuzzy dot d-ideal of X.

Theorem 4.5.

Let $f: X \rightarrow Y$ be an epimorphism of d-algebras. If $\mu^{f}$ is a fuzzy dot d-ideal of X. Then, $\mu$ is a fuzzy dot d-ideal of Y.

Proof. Let $f: X \rightarrow Y$ be an epimorphism of a d-algebras, and let $\mu^{f}$ be a fuzzy dot d-ideal of $\mathrm{X}$. Then, we have to show $\mu$ is a fuzzy dot d-ideal of a d-algebra $X$.

1. Let $a \in X$ and $b \in Y$. Then, $\mathrm{f}(\mathrm{a})=\mathrm{b}$.

Now

$$
\begin{aligned}
& \mu(b)=\mu(f(b))=\mu^{f}(a) \leq \mu^{f}\left(0^{\prime}\right) \\
& =\mu(f(0))=\mu\left(0^{\prime}\right)
\end{aligned}
$$

Hence $\mu(0) \geq \mu(b)$ for $b \in Y$.

2. Let $c, b \in Y$. Then there exists $a, b \in X$ such that $f(\mathrm{a})=\mathrm{c}$ and $\mathrm{f}(\mathrm{b})=\mathrm{d}$. 


$$
\begin{aligned}
& \mu(c)=\mu(f(a)) \geq \min \left\{\mu^{f}(a * b), \mu^{f}(b)\right\} \\
& \quad=\min \{\mu(f(a) * f(b)), \mu(f(b)\}=\min \{\mu(c * d), \mu(d)\} \\
& \geq \mu(c * d) \cdot \mu(d) . \\
& \text { Hence, } \mu(c) \geq \mu(c * d) \cdot \mu(d) \text {, for all } c, d \in Y \text {. } \\
& \text { 3. Let } \mathrm{f}(\mathrm{a})=\text { cand } \mathrm{f}(\mathrm{b})=\mathrm{d} \text { bi in Y. Then, } \\
& \mu(c * d)=\mu(f(a) * f(b))=\mu(f(a * b)) \\
& =\mu^{f}(a * b) \geq \min \left\{\mu^{f}(a), \mu^{f}(b)\right\} \geq \mu(c) . \mu(d) . \\
& \text { Hence, } \mu(c * d) \geq \mu(c) \cdot \mu(d), \text { for all } c, d \in Y . \\
& \text { Thus, } \mu \text { is a fuzzy dot d-ideal of Y. }
\end{aligned}
$$

\section{Discussion}

In this paper, we introduce the new concepts of fuzzy dot d-subalgebra as extension of a fuzzy d-algebrs and this will lead for future development of the theory of algebra and its application.

\section{Conclusion}

In this paper, the concept of fuzzy dot d-subalgebra has been introduced. Different characterizations have been investigated. We have also proved the equivalent of d-subalgebra and fuzzy dot d-subalgebra, different illustrations on fuzzy dot d-ideal has been introduced. As a future work, we will do on fuzzy commutative d-subalgebra.

\section{Acknowledgements}

The author of this paper forwards great thank to the referee for their genuine comments.

\section{References}

[1] Ahmed, B. (1993). Fuzzy BCI-algebras. J. Fuzzy Math, 2(1993), 445-452.

[2] Akram, M. and Dar, K. H. (2005). On fuzzy d-algebras. Journal of Mathematics, Vol. 37(2005), pp. 61-76.

[3] Bormmandseid, et al. (2016). Some connection between BCK-algebrasand n-array blockcods. arxiv: 1608.0368401, 2016, 1-9.

[4] Husein Abbas and Shukur Al-aeasbi. (2016). On fuzzy implicative ideal aBH-algebra. 2016, 59-70, Doi: 10.9790/5728-1704055970.

[5] Iseki, K. and Tanaka, S. (1976). Ideal theory of BCK-algebras. Math. Japonica, 21(1976), 351-366.

[6] Jie Meng. (1997). Young one Jun and HeeSij Kim, Fuzzy implicative ideals of BCK-algebras. Fuzzy Sets and Systems, Vol. 89(1997), 243-248.

[7] Neggers, J., Jun,Y. B., and Kim, H. S. (1999). On d-ideal in d-algebras. Math. Slovaca, Vol. 49(1999), pp. $243-251$.

[8] Neggers, J. and Kim, H. S. (1999). On d-algebra. Math. Slovaca, Vol. 49(1999), 19-26.

[9] Tapansenapati, et al. (2014). Fuzzy dot subalgebras and Fuzzy dot ideals of Balgebras. Journal of uncertain system, Vol. 8, No. 1(2014), pp. 22-30.

[10] Xi, O. G. (1991). Fuzzy Bck-algebras. Math. Japon., 36(1991), 935-942.

[11] Zadeh, L. A. (1965). Fuzzy sets. Information Control, 8(1965), 338-353. 